\title{
Covered self-expandable metal stent placement for post-endoscopic sphincterotomy bleeding in a patient with surgically altered anatomy
}

Although balloon enteroscopy-assisted endoscopic retrograde cholangiography (ERC) is a useful procedure for biliary intervention in patients with surgically altered anatomy, advanced skills are required. Therefore, if post-endoscopic sphincterotomy bleeding occurs during the procedure, endoscopic hemostasis is challenging. Placement of a covered selfexpandable metal stent (CSEMS), which has been reported as a useful hemostasis treatment for refractory bleeding after endoscopic sphincterotomy on conventional ERC [1-4], may be effective even in such a difficult situation. However, to our knowledge, CSEMS placement for such bleeding events has not been reported under balloon enteroscopy-assisted ERC. Here, we report a successful case of hemostasis for post-endoscopic sphincterotomy bleeding by CSEMS placement using balloon enteroscopy in a patient with surgically altered anatomy. A 77-year-old man with a history of distal gastrectomy with Billroth-II reconstruction, developed cholangitis as a result of choledocholithiasis. For biliary drainage, a short-type single-balloon enteroscope (SIF-H290S; Olympus Medical Systems, Tokyo, Japan) was inserted to the duodenal papilla. Following biliary cannulation and guidewire insertion, endoscopic sphincterotomy was carried out. However, massive pulsatile bleeding occurred immediately after endoscopic sphincterotomy. Although balloon tamponade using a dilation balloon catheter was performed, the bleeding could not be stopped and gradually worsened ( $\triangleright$ Fig. 1 ). Therefore, a CSEMS $(10 \times 60 \mathrm{~mm}$, Niti-S SUPREMO; TaeWoong Medical, Seoul, Korea) was placed across the duodenal papilla to cover the bleeding point, with the bleeding ceasing just after the placement ( $\triangleright$ Fig. 2 , $\triangleright$ Video $\mathbf{1}$ ). There were no adverse events and a transfusion was not needed.

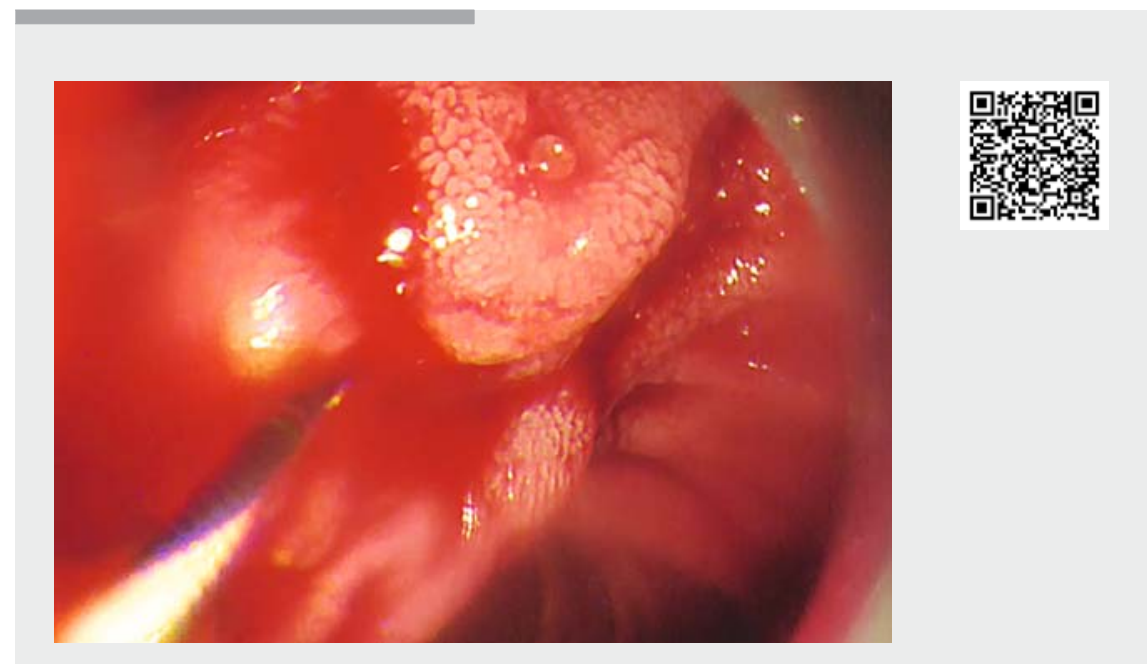

Video 1 Massive pulsatile bleeding occurred immediately after endoscopic sphincterotomy. Because the bleeding remained uncontrolled after balloon tamponade, a covered self-expandable metal stent was placed for hemostasis, after which the bleeding stopped.
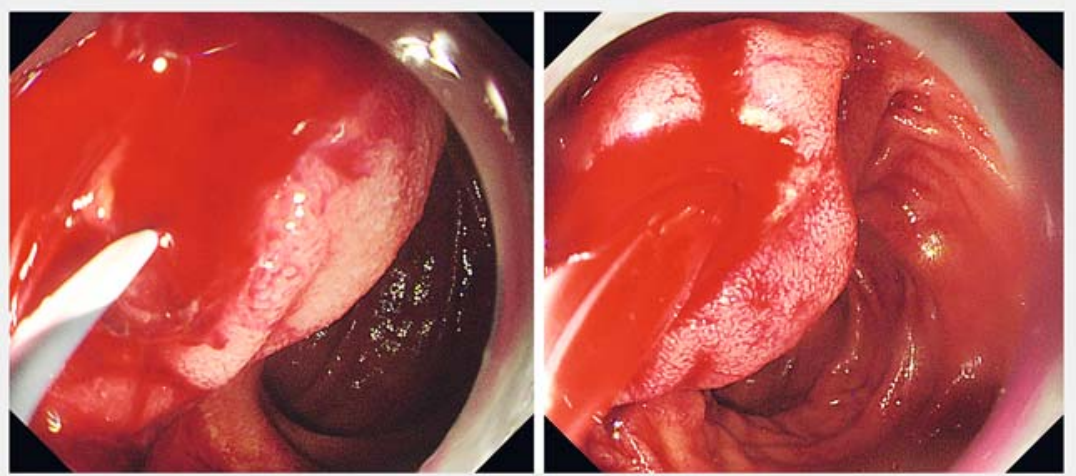

- Fig. 1 After the endoscopic sphincterotomy, massive bleeding occurred and remained uncontrolled after balloon tamponade.

The stent was removed 11 days after the procedure, and stone extraction was performed without any rebleeding.

Even under balloon enteroscopy-assisted ERC, a CSEMS can be placed and is effective for post-endoscopic sphincterotomy bleeding. The stent can also be removed without difficulty. CSEMS placement using balloon enteroscopy can be a useful rescue technique when massive and uncontrolled post-endoscopic sphincterotomy bleeding occurs in patients with surgically altered anatomy.

Endoscopy_UCTN_Code_CPL_1AK_2AC 


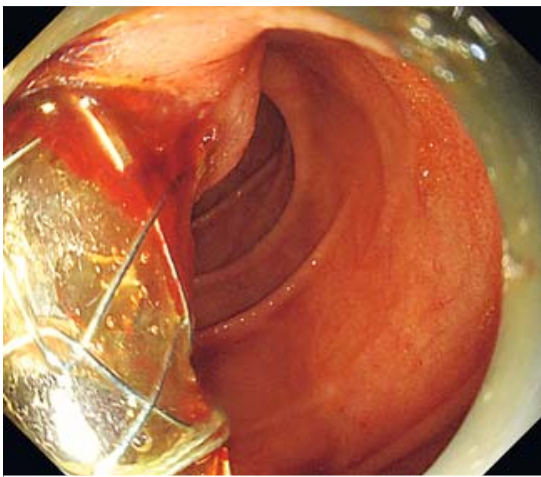

Fig. 2 A covered self-expandable metal stent was placed for hemostasis, after which the bleeding stopped.

\section{Competing interests}

None

The authors

Tadahisa Inoue, Mayu Ibusuki, Rena Kitano, Yuji Kobayashi, Kiyoaki Ito, Masashi Yoneda Department of Gastroenterology, Aichi Medical University, 1-1 Yazakokarimata, Nagakute, Aichi 480-1195, Japan
Corresponding author

\section{Tadahisa Inoue, MD, PhD}

Department of Gastroenterology, Aichi Medical University, 1-1 Yazakokarimata, Nagakute, Aichi 480-1195, Japan

Fax: +81-561-633208

tinoue-tag@umin.ac.jp

\section{References}

[1] Shah JN, Marson F, Binmoeller KF. Temporary self-expandable metal stent placement for treatment of post-sphincterotomy bleeding. Gastrointest Endosc 2010; 72: 1274-1278

[2] Itoi T, Yasuda I, Doi S et al. Endoscopic hemostasis using covered metallic stent placement for uncontrolled post-endoscopic sphincterotomy bleeding. Endoscopy 2011; 43: 369-372

[3] Canena J, Liberato M, Horta D et al. Shortterm stenting using fully covered self-expandable metal stents for treatment of refractory biliary leaks, postsphincterotomy bleeding, and perforations. Surg Endosc 2013; 27: 313-324

[4] Cochrane J, Schlepp G. Comparing endoscopic intervention against fully covered self-expanding metal stent placement for post-endoscopic sphincterotomy bleed (CEASE Study). Endosc Int Open 2016; 4: E1261-E1264

\section{Bibliography}

DOI https://doi.org/10.1055/a-0978-4815

Published online: 9.8.2019

Endoscopy 2020; 52: E13-E14

(c) Georg Thieme Verlag KG

Stuttgart · New York

ISSN 0013-726X

\section{ENDOSCOPY E-VIDEOS}

https://eref.thieme.de/e-videos

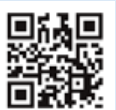

Endoscopy E-Videos is a free access online section, reporting on interesting cases and new techniques in gastroenterological endoscopy. All papers include a high quality video and all contributions are freely accessible online.

This section has its own submission website at

https://mc.manuscriptcentral.com/e-videos 